\title{
Research on Influence of Handicraft in Design History from the Diagrammatic Perspective
}

\author{
$\mathrm{Yu} \mathrm{Fu}$ \\ The Open University of China \\ Beijing China 100039
}

\begin{abstract}
Handicraft had gone from domination to the challenge of industrialization throughout the design development process and then it had been risen again after revolt. From domination to the challenge of industrialization throughout the design development process, handicraft had risen again after decline. Meanwhile, the influence of handicraft had changed along with a variety of factors. The development of design was analyzed according to the influence of handicraft based on its concept and characteristics in this paper. The change of influence of design, and its reasons were analyzed by Diagrammatic method. It is indicated that the key factors are industrialization and commercialization, and the essential factors are human values and human needs.
\end{abstract}

Keywords-Handicraft; Influence; Industrialization; Postindustrial society

\section{INTRODUCTION}

Nowadays, when humans freely enjoy the yielded fruitful results brought by modern civilization and industrialization, and consume the material wealth accumulated by industrial production, they pay a heavy price for the social crisis caused by physical property domination. People try to retrieve the lost things at one time, unearth national spirit, affective meaning, cultural attribute and human values in design from the concept of figure of handicrafts tradition. Therefore, handcraft revives to some extent and highlights the unique charm.

As a matter of fact, handcraft spans the long agricultural society and passes the industrial stage of collision with the large-scale production of machines, receives exclusion and resist circuitously. It is taken seriously when stepping into the postindustrial society. The author uses the perspective of the development and change of handcraft influence, reviews the process of design development and unearths the primary causes why handcraft, a production mode closely related to social formation, economic condition, productivity condition and cultural factors, can bring the above changes.

\section{THE CONCEPT OF HANDCRAFT}

Before discussing handcraft, first of all, we need to clarify the meaning of "craft". The word craft is quite old and it first appears in the literature in the 9th century [1]. Etymology thinks that the original "craft" is "ART"-skill. Japanese folk art theorist Soetsu Yanagi thinks: the early art is craft and skill. The concept of craft becomes independent when William Morris and Cobden-Sanderson clarify the differences [2] between Art and Craft in The Arts and Crafts Exhibition Society.

Craft derives from practical manufacture. Originally, all the crafts are finished by handwork. Handwork means making arts and crafts of unique artistic style with manual labor as the main body. It is a crafting skill with certain technique and artistry. The word "hand" stipulates the essential attribute of manual labor, refers in particular to arts and crafts different from using the method of big industrial mechanization to produce groups of standardized products. Although handcraft can be with the help of mechanical tools, the premise is that craftsman's direct manual work is still the main source of products.

\section{THE CHARACTERISTICS OF HANDCRAFT}

The characteristics of handcraft can be summarized as: the peculiarity of handcraft, the beauty of handcraft, the emotion of handcraft. So to speak, "Appealing design can be pleasing to the eye. Beautiful items are to be practical. Beautiful meaning affects the heart".

\section{A. The Peculiarity of Handcraft}

The peculiarity of handcraft not only derives from the attribute of manual labor itself, but also be under the influence of external environmental factors.

1) The unique attributes made by subjective factors. The traditional handcraft is a skill manifestation form based on manual labor and a combination of "skill" and "art". The rich creativity and variability of handcraft labor make handicraftsmen exert "intelligence". So each piece of handcraft is an independent process of art creation. It is the most unique and significant feature different from large machinery industrial production [3].

On one hand, because not every craftsman has the same level and sufficient time to engage in making goods. Products made by different craftsmen can show different features and levels. Then they are marked unique features by different producers and become the embodiment of handcraft uniqueness. On the other hand, because the improvement of handcraft production process, the inheritance of skills and the accumulation of experience depend on people and the whole process rely on the hands to complete. Even though the productions made by the same craftsman, they have some differences because of some factors and show some kind of uniqueness. Compared with machines of standardization 
production, human sensibility, subjectivity, creativity and imagination run through manual labor all the time and even the different state of mind, emotion and thought of craftsmen themselves can influence the hand feeling of make and let the order of importance and emergency is different when making the products. These differences always make products from the same craftsman have their own features. Each piece has uniqueness and it is impossible to clone.

2) The unique attributes made by objective factors. Kao Gong Ji, the earliest handcraft technology document existing in our country, makes incisive elaboration for handcraft creation view: "The day has changes of climate and seasons; the earth has different geographical condition; materials have beauty of their own; human have creativity and technology skills. If we combine four of them, we can make delicate design". "Exquisite workmanship" emphasizes the subjective factors and the rest three aspects belong to the objective influence.

Handcraft is based on nature. "Cai Mei" means a certain character of a kind of material is very suitable for making an artifact. The beauty of materials is a kind of physical property in essence, and can make the unique attributes of products from practical aspect or aesthetic aspect. Furthermore, traditional handcraft also pays attention to comply with the natural conditions and conform to producing area and geographical condition. "Tian Shi" means the restriction of natural conditions and also refers to that handcraft needs to adapt to the natural law proactively. "Di Qi" emphasizes the influence of original area and geographical condition. Different natural conditions and social customs of a place and climate promote the growth of different local crafts. Handcraft in different regions has strong local color and reflects different regional aesthetics.

\section{B. The Beauty of Handcraft}

The beauty of handcraft mainly embodies in three aspects such as the beauty of utility, the beauty of human nature and the beauty of decoration, etc.

1) The beauty of utility. Looking from history, craft originates in practical production. Moreover, the inheritance and development of handcraft as well take the basic idea of "making the best use of everything". In traditional social relations, handicraftsmen undertake social functions of providing and making necessities of life for food, clothing, housing and transportation and they are a kind of effective social productivity. In the development history of craft, the thought of attaching great importance to the function can be seen everywhere. Wang Fu in Han dynasty thinks "all sorts of workmen should pay the most attention to utility and attach the least importance to decoration"; Li Yu in Qing dynasty once says "items made by people must ensure that everyone can prepare and every family can use" [4]. Therefore, the beauty of utility is the source of the survival and development of handcraft. "Use" means serving the life and if craft leaves "use", it can't have advanced "beauty".

2) The beauty of human nature. According to the opinion of Soetsu Yanagi, because the groups served by handcraft are different, it can be divided into aristocratic craft and demotic craft (that is Min Yi). "Min Yi" means folk handcraft made by craftsman of folk handcraft. They are handcraft products or utensils used by ordinary people in daily life [5]. One of the characteristics of handcraft is that it is passed on from generation to generation under similar life forms. And the leading role in the inheritance-person become the major factor [6]. In handcraft, person is not only the starting point, but also the foothold. Handcraft can endow physical property with humanity. Handcraft is based on the needs of people and relies on people to develop and people's hands to work and manufacture. Human thoughts flow on fingertips. The accumulation and inheritance of production experience are full of wisdom. Craftworks permeate human feelings and eventually serve people's life. Handcraft combines creative thinking of craftsman and their feelings toward life; understanding of culture delivers humanistic care, cultural connotation and the respect towards personality, all showing the beauty of human nature.

3) The beauty of decoration. Exquisite handcraft can endow articles with elegant appearance and add the beauty of decoration to practical handcraft products. Essentially speaking, first of all, the beauty of decoration should depend on the practicability of utensils. If the beautiful decoration leaves the practicability, it is nothing else but attending to trifles and neglecting the essentials. Therefore, if we want to gain the beauty of decoration, we must start from the combination of deep aesthetic with "utility". Besides, the beauty of decoration comes from the special attribute of handcraft. It makes every utensil have its own features and can convey cultural connotation, bear the national spirit and the social symbolic significance.

\section{The Emotion of Handcraft}

Handcraft endows articles with rich spiritual value and emotional connotation through manual work and makes products become the medium for the communication between spirit and aesthetic; it congeals wisdom into precision carving with the body temperature of skillful craftsmen and delivers silent and deep feelings. This embodies the profound connotation of handcraft: creation meets the needs of survival; sculpt comes from aesthetic motivation; design includes contents such as utility, aesthetics, emotion, concepts and individuation. Therefore, it doesn't have the panic of industrial stage and the separation of design and production because of detailed division of labor, and the tedium and indifference caused by standardized mass production and let alone all kinds of embarrassment of circling round between balance and compromise.

\section{ANALYSIS ON THE CHANGES OF HANDCRAFT INFLUENCE}

Design depends on production mode and production mode reacts on design style and it is one of the important dynamics for promoting design development. Throughout the process of design development, handcraft experiences the whole process of ruling all the land, gradually being elbowed out, resisting with twists and turns, fading away and then returning to people's sight. During this time, the influence of 
handcraft has changes of ups and downs because of the effects of various factors "Fig. 1". These changes are the results of joint action of many factors. The schematic diagram comprehensively presents the evolution of the social formation, productivity condition, the division of design stages, design movement and design style, and adds the time of industrial revolution and the factors associated with handcraft to it. It intuitively analyzes and summarizes the situation changes of handcraft influence.

The schematic diagram researches handcraft in two processes of "social formation" and "design stage" from horizontal angle. After civilization coming into being, social formation can be divided into agricultural society, industrial society and post-industrial society successively [7]. Even though the evolution direction of design and the changes of social formation are basically the same, they are not one to one correspondence. The schematic diagram divides the design stage into pre-modern period, modernism period and post-modern period. The post-modern period basically corresponds with post-industrial society. Modernism period is the product of the golden age of industrial society.

The schematic diagram uses dark stripes and light color block to show the influences of handcraft and industrialization respectively from vertical perspective and intuitively show the variation tendency of handcraft influence.

\section{A. Ruling All the Land}

Handcraft becomes mature gradually with the development of human beings. Manual labor accompanies human beings through the wild primitive period and creates the first civilization form-agricultural civilization. Humans begin the technical history when they are self-sufficiency [2]. In the agricultural society, the survival and development of handcraft have close relationships with the lifestyle and economic conditions at that time. Firstly, manual production level depends on human factor. Secondly, the work of scattered workshops style in agricultural society influences the spread of handcraft to a certain degree. Thirdly, feudalism makes the handcraft mode under protection. Craftsmen often accept orders from feudal lords and let handcraft serve the nobility. Fourthly, relatively closed environment and limited communication make the transmission of handcraft production experience is limited to small range and it even inherits only within the family. Fifthly, the consummate handcraft skills must depend on hands-on practical exploration and long-term accumulation to acquire. The acquisition of this kind of individual experience cannot be duplicated in a large scale. This shows that in agricultural society, handcraft almost occupies the dominant position in the long run with stable development and relatively complete system "Fig. 1-A".

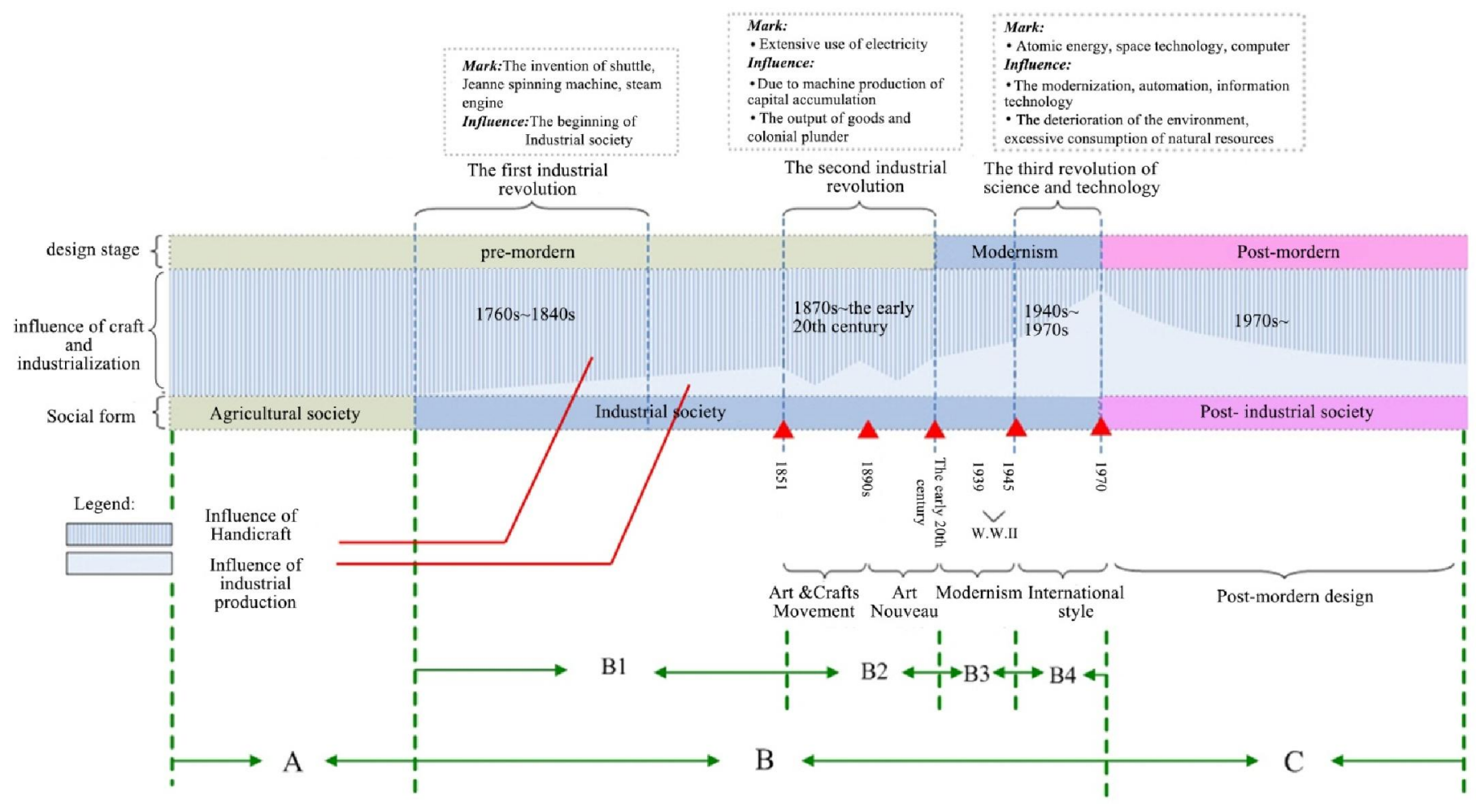

Fig. 1. diagram of Influence on Changes of Handicraft 


\section{B. The Road with Twists and Turns}

Before the industrial revolution, manual operation is the only production mode occupying the dominant position. From the first industrial revolution to 1970 s, handcraft suffers the powerful challenges from industrialization. And the handcraft influence presents the trend of obvious shrinking decline on the whole "Fig. 1-B". Of course, during this time, handcraft and industrialization have inversely proportional relationship and fight circuitously. The influence of handcraft turns from strong to weak, being elbowed out, fading away and then becoming stronger. The twists and turns during this period are the reverse motion, exploration, thought and reestablishment of various forms of handcraft.

1) Handcraft begins to decline. The first industrial revolution overturns the basic pattern of agricultural society and brings people to industrial society. The situation of handcraft ruling all the land was broken "Fig. 1-B1". The arisen mechanical industry brings high efficiency, large production and low cost. The new instruments of production have differences in essence from the past instruments in handcraft industry. It turns the principal part of production from subjective perceptual person to objective rational machines. Art critic Herbert Read points out the real difference between the two: a person uses his hands to use tools to make articles. This article shows the tendency of his will and traces of his personality in every stage of production; while machines can produce precise products in uniformity without the interferences of person. These products don't show unique changes and don't have the charm of personality as well [8]. Industrialization fiercely impacts the handcraft in rude way and urges people and machines to combine with each other. The engineering industry of mechanization, functionality and quantity production will eventually replace manual labor on a large scale and destroy the principal part of handcraft (that is folk art) and make design link independent from production. Engels says: "the reason why the newborn industry can grow up like this is that it uses machines to replace hand tools and uses factories to replace workshops..."

2) The resistance and renaissance of handcraft. The impact and replacement that machines do to handcraft don't accomplish in an action. The several industrial revolutions have different levels of influences on handcraft respectively and trigger multiple design movements in the name of revitalizing handcraft. In general, handcraft revolts mainly in three ways: the first one is various types of handcraft renaissance movement. From the mid-19th century to the early 20th century, many countries and regions successively experience arts and crafts movement, art nouveau movement and decorative art movement, etc. which can be regarded as the rebellion and resistance of handcraft against the three industrial revolutions. They pretend propositions from the aspects such as labor form, aesthetic style, decorative style and the class nature of design, etc. The second one mainly refers to that traditional handcraft still can firmly exist in the appreciative craft (which are personal craft and noble craft).
The third one is that because of the limited impact from industrialization, handcraft still can maintain the basic complete structure in areas with underdeveloped productivity.

To be specific, in the period of industrial society, the resistance and renaissance movement of handcraft intensively appear during the second industrial revolution. Compared with the first industrial revolution, the second one has larger influence, more broad impact scope and spread faster. It accelerates the industrialization process objectively and makes the confrontation between handcraft and industrialization more intense.

The year 1851 is the first historical turning point "Fig. 1B2". The arts and crafts movement originated in Britain is the first design movement which has widespread impact after entering the industrial society. It is clearly against machine production, showy and not substantial, and highly praises the sincere design of combination of art and handcraft, shows the resistance that the society which regards handcraft as the main part does to newly developed industry. Because handcraft occupies the dominant position of the production mode in the long term, its influence is still the leading force and the initial power of industrialization is limited, it certainly will be strongly pressed and confronted by handcraft. Even though the movement with Utopia color eventually ends in failure, the ideas such as combination of art and handcraft, utility and beauty, serving the public advocated by it highlight the advantages of handcraft and delay the impact that mechanized production has on handcraft objectively. However, for traditional handcraft, this turning point after all declares that a new era is around the corner.

In the end of 19th century, the art nouveau movement which inherits many thoughts of arts and crafts movement comes on stage "Fig.1-B2". It has bigger influence and its scope spreads throughout Europe and America and even the international world. It uses the proposition of advocating nature to oppose industrialization, aiming at attracting people's attention for traditional handcraft again. But in essence, the art nouveau movement inevitably takes the double meanings of connecting link [9]: it not only takes the high praise, renaissance and reminiscence for traditional handcraft, but also inevitably reflects the requirements of the new industrial age. Among them, the design of Vienna Secession, Jugendstil and Glasgow School begin to have utterly different ethos and become the omen of modernism design. Even though the above two movements have distinct intention of revitalizing handcraft, they are seemed increasingly powerless in front of productivity revolution. The subsequent decorative art movement simply begins to envisage industrialization and directly adopts compromising methods and combines dual characteristics of handcraft and industrialization to design. William Morris says: "in this period, people always have the will to fully regain the handcraft manufacturing methods; but it is worth thinking that this kind of will to what degree is just a vent of emotion with powerless resistance for the reality. Meanwhile, to what degree it is an omen of the real and upcoming revolution in our living habit. Just like the past revolution which has given rise to the mass production system of mechanization, it is irresistible as well [10].” 
In this process, handcraft spares no efforts to maintain its dominant position, starts different forms of resistance. Each change is trying to slow down the impact of industry. But each time it becomes weaker. All the efforts after all cannot stop the irresistible trend of productivity. And the acting force of handcraft renaissance gradually fades away.

3) The accelerating decline of handcraft. Morris has to admit: "as a kind of living condition, mechanized production is completely a sin; but as a method that still has and sometimes created more ideal living conditions for us, it is also essential. [8]" The year 1945 is also another turning point "Fig. 1-B3", and hereafter industrialization shakes and destroys the status of handcraft with a further intensified tendency: modernism design movement and internationalism makes folk art, the main part of the past handcraft, be fully replaced by industrialization; personal and aristocratic craft suffer a huge impact with the advance of the democratic process of design. At this time, handcraft exists in the form of extremely marginalization and lurking and its overall situation depends on a thread.

However, according to the analysis we can know that the appearance of this kind of situation has certain inevitability: first of all, compared with manual technology, the industrial production is undoubtedly progressive. It conforms to the inevitable trend of historical development. Secondly, after the failures of several handcraft renaissance movements focusing on escaping from reality, the step of industry doesn't stop. On the contrary, new technology and new equipment emerge one after another. They are in urgent need of the design matching with them to settle realistic problems. Thirdly, the economic development gives rise to great changes of social ideology and culture. In the first half of the 20th century, the Europe and America spring up the anti-traditional ideological movement and the fields such as philosophy, aesthetics, art, literature, drama, music and psychology raise the modernism trend of thought simultaneously. Fourthly, in terms of design, the handcraft has obvious meaning to serve the nobility. And one of the purposes of modernism design is to solve the problem of the social function of design, strive to serve the public through design and reflect new design concept: advocating geometric modeling which gives priority to function is extremely suitable for the requirements of standardized mass production. "The decoration is abandoned by designers of the new generation. They try to update the design language according to the challenges and requirements of the era. These challenges and requirements come from technology, aesthetics, economy, politics and culture as well [11]." Therefore, when industrial production and its aesthetics and attributes are popular as the opposite side of handcraft, the uniqueness, aesthetic perception and human nature of handcraft fully experience the cold treatment.

The third technological revolution and the World War Two make modernism develop into international style. It extremely stresses the beauty of machine and rationalization and totally denies decoration and completely makes form and function become opposite, and makes modernism become one-sided and extreme, turns design into elitism and pushes the influence of industry to the peak "Fig.1-B4". Sullivan uses "forms following function" to point out the key spirit of functionalism;
Meath turns internationalism into equivalent formalism through "less is more, even pursuing manimum through violating the function". Adolf Loos puts forward "refusing decoration, people will become more and more civilized" in the Ornament and Crime, totally denies decoration and as well denies handcraft and its aesthetic ideas. Internationalism regards handcraft as the pronoun of backwardness and thinks handcraft is contrary to modern civilization. Loos insists that "the most mature society need to express through the most concise form. And the designers' duty is to insist this principle... Decoration is the omen of backwardness and degradation. The decoration made by handicraftsmen means great waste of labor, materials and capital." Thus handcraft hits the floor. The aesthetic ideas, ideology and culture and class status represented by it are regards as heresies.

\section{Returning to People's Sight}

This kind of situation changes until the post-modern period "Fig. 1-C". In 1960s, on one hand, the pluralistic economy expands. The materials accumulated by industrialization are unprecedented rich. The product similarity sharply increases. All of these trigger the reflection of design. On the other hand, material prosperity and industrial development bring disadvantages. In 1970s, many western countries widely encounter economic crisis, which causes contradictions such as politics, culture and psychology, practical problems such as ecological crisis, energy crisis, moral crisis, etc. leading the internationalism to move toward the dead end. The postmodern design begins to rebel and surpass, and shows the regression of spirit and history of handcraft: on the technique, it all or partly adopts handcraft style and historical elements to design; on the concept, it anew picks up the design concept and aesthetic rule abandoned by modernism - use "less is annoying" to replace "less is more"; it uses deconstruction and duplication to question the authority; it anew stresses artistry through decoration; it uses ironic and joking techniques to overturn the design classics of modernism; it uses ambiguous multiple metaphor to oppose dualism; it uses sensibility and sexuality to oppose rational supremacy; it uses unique design modality to manifest individuality and express humanistic care and presents the tendency of revitalizing handcraft spirit. The proposition of revitalizing handcraft spirit can receive mass response. It is not only because internationalism is widely questioned, but also because the post-modernity pays increasing attention to human nature, individuality and human needs and it attaches more importance to human-oriented values. The post-modernity is the change of design style as well as the introspection of design concept. The handcraft spirit "pays attention to the heart and can give the joy of labor, make people abide by the moral and it is also factor endowing the articles with attribute of beauty. Therefore, handcraft work can also be called the work of heart. [12]" It happens to hold the same views with many propositions of post-modernity. The shimmer of handcraft eventually uses the unique way to shine into the post-modern reality hundred years later. Its important value and influence finally anew win the affirmation.

\section{CONCLUSION}

This shows that the critical factors deciding the change of handcraft influence can be classified as industrialization and 
commercialization. But in essence, it depends on the cognition towards "human value" and "human needs":

As shown in the figure, every change of handcraft is almost directly under the influence of industrialization. Industrialization is always seen as the opposite side of handcraft. They resist and compete and have inversely proportional relationship. But instead of saying that this kind of challenge comes from industrialization, we say it stems from the revolution of productivity.

If we say industrialization is the direct reason affecting the development of handcraft, then commercialism is the catalyst which speed up the change. Mr. Hume thinks: as "the craft of capital", it "never misses any production opportunity allowed by business ideas. [1]" Essentially speaking, the commercialization itself is a neutral element. Consumption and market not only objectively reflect the disadvantages of the insufficient handcraft production and aristocratic tendency, embody the superiority of industrial production, but also make industrial production become more eager for quick success and instant benefits, intensify more penetrating contradiction and promote the design change.

In conclusion, this article bases on the angle of view of the changes of handcraft influence, and reviews the process of design development. Essentially speaking, manual operation and mechanical industry are two different methods of manufacturing and represent different levels of productive forces. However, the most basic reason deciding the change of handcraft influence lies in: both of them contain different ideology, values and design philosophy - that is the cognition of "human value" and "human needs". Although handcraft is less efficient than machine, it has natural attributes and can highlight human nature, deliver emotions, create the uniqueness and artistic aesthetic feeling of products. It is a production mode with people as the dominant force, always affirms human values, attaches importance to human needs and put emphasis on human role. When industrialization increases efficiency rapidly, reduces the cost and enriches the materials, it gradually turns to functionalism, mass production and materiality as the core and sets up aesthetic rule matching with it. Even though the rational concept focusing on function and opposing decoration are important assurances to realize democratization of design, the concept starting from physical property makes industrial production inevitably have the color of utilitarian and neglects the subjective demands and feelings of people to some extent. No wonder Soetsu Yanagi says "at least now, machinery has been shocked by many unnatural and inhuman elements. If machinery can operate normal production, it as well can return to the pathway of nature and humanity and then appears with a new attitude. What we should not neglect is the essential factors in manual nature and they are not influenced by time. [2]"

\section{REFERENCES}

[1] Du Junhu. Discuss on the Two Mistakes of Current Handcraft Research [J]. Hundred Schools in Arts, 2006,(2):86-89.

[2] Soetsu Yanagi (Janpan). Craft Culture [M]. Nanning: Guangxi Normal University Press, 2011.
[3] Chen Jun. The Cultural Inheritance of Traditional Handcraft and Contemporary "Redesign" [J]. Literature\&Art Studies, 2012,(5):137139.

[4] Wang Minghui. Brief Analysis on Chinese Craft Design Idea [J]. Art and Literature for the Masses, 2011,(6):44-45.

[5] Zhang Sunchen. About the Exploration of Soetsu Yanagi's Folk Art Thoughts [J]. Hundred Schools in Arts 2011, (8):13-314.

[6] Hang Jian. The Thoughts of Handcraft [M]. Jinan: Shandong Pictorial Press, 2001.

[7] Chen Xiaohua. Between Craft and Design [M]. Chongqing: Chongqing University Press, 2007.

[8] Periodicals Editorial Board. Technical Aesthetics and Industrial Design Periodicals [M]. Tianjin: Nankai University Press, 1986.

[9] Wang Shouzhi. History of World Contemporary Design [M]. Beijing: China Youth Press, 2002.

[10] Li Yanzu. Selected Readings of Classic Works of Foreign Design Art [M]. Beijing: Tsinghua University Press, 2006.

[11] Penny Sparke (Britain). Introduction to Design and Culture [m]. Nanjing: Yilin Press, 2012.

[12] Soetsu Yanagi (Japan). Japanese Handcraft [M]. Nanning: Guangxi Normal University Press, 2006. 